\title{
DEPENDENCY OF THERMO-PHYSICAL PROPERTIES ON SURFACE BONDING OF BN NANO-CRYSTAL
}

\author{
BHOOPENDRA DHAR DIWAN* \\ Department of Basic Sciences, Dr C V Raman University, \\ Bilaspur 495001 Chattisgarh, India \\ Email: bddiwan@gmail.com ${ }^{\ddagger}$ \\ N. KUMAR SWAMY \\ Material Science Research Lab, ITM University, \\ Gurgaon 122017 Haryana, India \\ Email: nkumarswamy15@gmail.com
}

\begin{abstract}
In this paper we have studied the variation of normalized per-atom pair cohesive (binding) energy and melting temperature with size and number of atom-pairs in the BN nano-crystal by simple model approach. In small nano-particles, large proportions of their atoms reside either at or near the surface, and those in clusters are basically all on the surface. So, we have to study the surface configuration in detail that leads the constancy of the crystals. Even for bulk materials, parameters that ensure this constancy involving normalized cohesive energy, surface reconstruction, iconicity, bulk structure, hybridization and charge balance, melting point. It is worthwhile to say that many of these factors are quite interdependent. These all factors influence the whole cohesive energy of crystals and give important information about the deflections from inverse size dependence. The per-atom-pair binding energy and melting temperature of BN nano-crystal is a quadratic function of the inverse of the crystal size. The binding energy and melting temperature comes near their bulk value with increasing the crystal size and same as the bulk material when the crystal size is above than $100 \mathrm{~nm}$.
\end{abstract}

Keywords: Size effect; binding energy; Melting temperature; Nano-crystals; Nano-cluster.

\section{Introduction}

In the last decade more and more focus has been set on studying the thermodynamic properties of nano-sized materials due to their wide application in fields such as microelectronics, nonlinear optics and solar energy. Boron nitride (BN) is significant materials among the other semiconductor compounds because of its highly desirable thermal, mechanical, electrical, and optical properties; therefore it is used for safety coating films, high-power and advanced microelectronic devices at high temperature. ${ }^{1}$ In general cubic boron nitride is the hardest material than other material (accept diamond)

\footnotetext{
"Bhoopendra dhar Diwan. Bilaspur (C. G.) India.

${ }^{\dagger}$ Department of Basic Sciences, Dr C V Raman University, Bilaspur 495001 Chattisgarh, India. "bddiwan@gmail.com.
} 
and a similar indirect electronic wide-band gap semiconductor. ${ }^{2}$ Among these properties, cohesive (binding) energy is the most practical property in nano- crystals applications because it determines the other physical properties such as vapor pressure, melting point etc.. Number of investigation based on the assumption that the cohesive (binding) energy of nano- crystals are regulated by nearest neighbor interactions, which leads to a linear dependence of this cohesive (binding) energy on the inverse size of the crystals. ${ }^{3-6}$ This assumption is reasonably good for many metals both single and ensembles crystals founded entirely on the bulk cohesive (binding) energy and the size distribution. Nevertheless, for non-metallic semiconductor nano-crystals where both covalence and iconicity play a significant part in the bonding, the assumption is no more valid. Hence, in order to better interpret the basic physical properties of small-scale semiconductor materials we need to do a theoretical study of their cohesive (binding) energies as a function of their material size. In this way can find out their deals with their detailed atomistic bonding.

$\mathrm{Li}$ et al. ${ }^{7}$ have successfully synthesized nano-scale BN by using encapsulation method and detected strong photoluminescence. Oku et al. ${ }^{8}$ have synthesized the BN fullerene materials such as nano-tubes, nano-capsules and nano-cages by using an arc-melting method. Liu et al. ${ }^{9}$ have investigated the structural stability and electronic properties of $h$ BN by first-principles calculations based on the density-functional theory local-density approximation. Cai et al. ${ }^{10}$ have studied the dielectric and vibrational properties for $h-B N, c-B N$ and $w-B N$ by using first-principles. Knittle et al. ${ }^{11}$ have studied the phase diagram of $\mathrm{BN}$ by experimentally (X-ray diffraction) and theoretically (first principle method). Goldstein et al. ${ }^{12}$ have studied the size dependence melting temperature of CdS nano-crystals and demonstrated experimentally that when one or more dimensions of the semiconductor crystal are reduced to a size comparable to bulk electron delocalization lengths (tens to hundreds of angstroms). They also show that a larger fraction of the total number of atoms is on the surface of crystal. Farrell et al. ${ }^{13}$ have studied that the per atom pair binding (cohesive) energy of small nano-particles and compound semiconductor clusters is a quadratic dependence of the particle diameter.

Moreover, the model calculations can now be executed comparatively easily for the determination of many relevant physical properties, especially in the small-scale range where experimental findings are not easy. The possibility of investigating the overall behavior of nano-crystal and cluster depending on its size variation has attracted us to work on this paper.

\section{Method of Computation}

According to our model approach, the binding energy per atom pair $\left(E_{n b}\right)$ of nano-crystal can be estimated from the relation as,

$$
E_{n b}=E_{b}\left(1-1 / n^{2}\right)
$$


Where, $E_{b}$ is the binding energy for the bulk crystal and $n$ is the cube root of the total number of atom-pairs in the crystal. ${ }^{13}$ Here we will take $d \mathrm{~nm}$ is the diameter of nanocrystal than we get the volume of the nano-crystal is

$$
V_{\text {Total }}=4 \pi(d / 2)^{3} / 3
$$

and the volume of a single unit cell for the nano-crystal is

$$
V_{\text {unit }}=a^{3}
$$

Here $a$ is the lattice constant of the unit cell. Therefore the total number of unit cells in nano-crystal is as

$$
N_{\text {unitshell }}=V_{\text {total }} / V_{\text {unit }}
$$

In the face centered cubic crystal, the number of atom pair in unit cell is 2 , therefore the cube root of the total number of atom-pairs in the particle is

$$
n=\sqrt[3]{2\left(V_{\text {total }} / V_{\text {unit }}\right)}
$$

Here it is noteworthy that the bulk melting point is proportional to their binding energy for most of semiconductor compounds. ${ }^{13}$ Thus having a similar assumption for the corresponding nano-crystal, we can write

$$
T_{m n}=T_{m}\left(1-1 / n^{2}\right)
$$

or

$$
T_{m n}=T_{m}\left[1-\left(r_{0} / r_{n}\right)^{2}\right]
$$

Here $r_{0}$ is the average radius of an $n=1$ particle and $r_{n}=n r_{0}, T_{m}$ and $T_{m n}$ is the melting point of bulk and nano-crystal, respectively. The average distance $\left(r_{0}\right)$ for face centered cubic crystal can be defined as

$$
r_{0}=\left(V_{\text {unitcell }} / 2 Z\right)^{1 / 3}
$$

\section{Results and Discussion}

The input parameters such as lattice parameter $(a)$, cohesive (binding) energy per atom pair $\left(E_{b}\right)$ and melting temperature $\left(T_{m}\right)$ of BN bulk crystal are reported in table 1 .

Table 1. Input parameters $a(\mathrm{~A}), T_{\text {bulk }}(\mathrm{K})$ and $E_{b u l k}(\mathrm{eV})$ for cubic BN bulk crystal.

\begin{tabular}{cccc}
\hline Material & $a$ & $T_{\text {bulk }}$ & $E_{\text {bulk }}$ \\
\hline $\mathrm{BN}$ & $3.618[11]$ & $3246[11]$ & $13.5[11]$ \\
\hline
\end{tabular}


The calculated normalized per atom-pair binding energies of BN nano-crystal are shown in figure 1-2. It is clear from figure 1-2 that the per atom-pair binding energies diverge from a linear dependence on $1 / n$ (or $1 / r_{n}$ ) and show an approximately quadratic dependence on $1 / n$ (or $1 / r_{n}$ ) for BN nano-crystal. As antecedently observed, this behavior is counterpoint to the linear dependence determined for most metals. ${ }^{13}$ The cause for this fluctuation in size dependence on the covalent nature of the bonding in semiconductor materials as well as low aspect-ratio metals. The point on the far left and right in figure 1 are concerned to the bulk material $(n=\infty)$ and single atom-pair $(n=1)$.

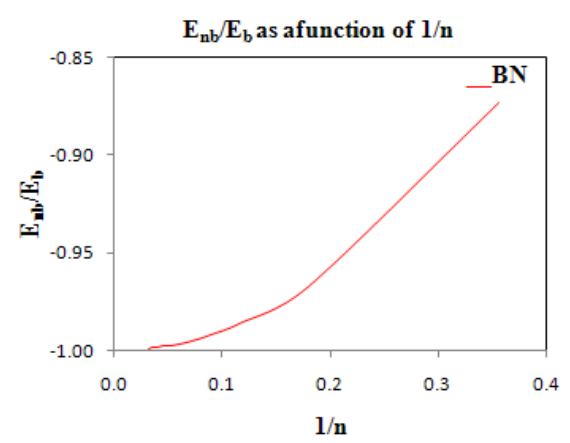

Fig. 1. Variation of relative cohesive energy as a function of $1 / \mathrm{n}$ for BN nano-crystal.

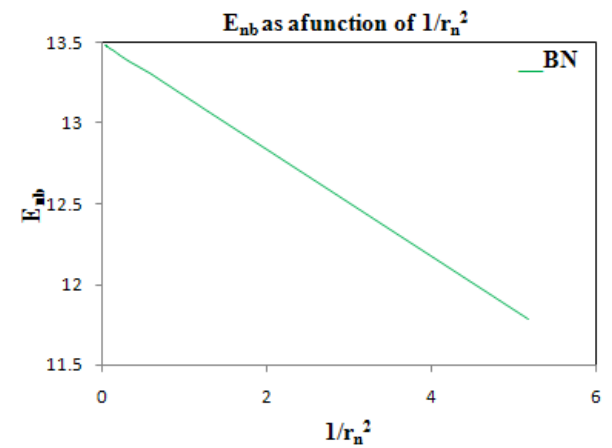

Fig. 2. Variation of cohesive energy as a function of $1 / \mathrm{r}_{\mathrm{n}}^{2}$ for BN nano-crystal.

We know that the cohesive energy of crystal reveals the strength of metallic bond. Our result shows that, decrease in the cohesive energy of nano-particle means decrease in the strength of the corresponding metallic bond. So it will be easier to break the metallic bond, which will lead to decrease in the melting point of nano-crystal. The calculated melting temperature $\left(T_{m n}\right)$ of $\mathrm{BN}$ nano-crystal is shown in figure 3-4. Figure 4 displays the variation of melting temperature $\left(T_{m n}\right)$ as a function of $1 / r_{n}^{2}$ for BN nano-crystal. It is clear from figure 3-4 that the melting temperature $\left(T_{m n}\right)$ diverge from a linear dependence on $1 / n$ (or $1 / r_{n}$ ) and are a close to quadratic dependence on $1 / n$ (or $1 / r_{n}$ ) for BN nano-crystal.

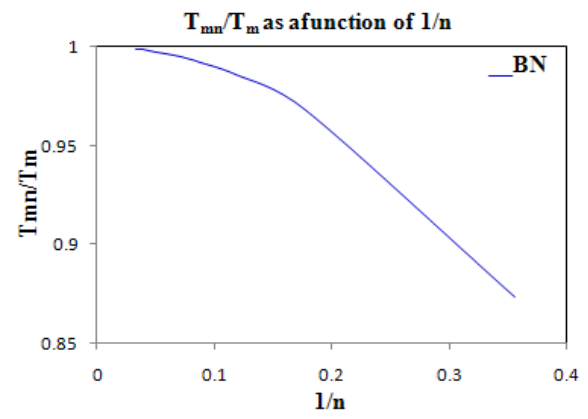

Fig. 3. Variation of melting temperature as a function of $1 / \mathrm{n}$ for BN nano-crystal.

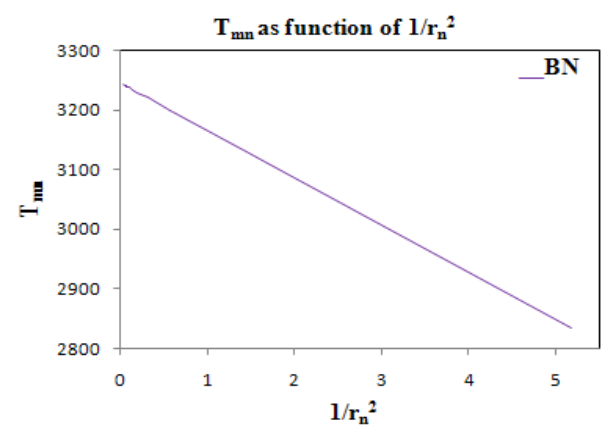

Fig. 4. Variation of melting temperature as a function of $1 / \mathrm{r}_{\mathrm{n}}{ }^{2}$ for BN nano-crystal. 


\section{Conclusion}

An overall assessment shows that the description of the per-atom pair cohesive (binding) energy and melting temperature of $\mathrm{BN}$ nano-crystal attained by us is noteworthy in view of the inherent simplicity of the simple model approach. With all this, the model can be considered as the triumphant approach to explore a variety of factors that influence the cohesive (binding) energies and stability of small-scale and cluster semiconductor. It can be observed that the reports are well fit by a straight line in this size range rationalizing our assumption of a close to $1 / n^{2}$ or $1 / r_{n}^{2}$ dependence. We hope that the findings of this paper will certainly stimulate the work on the $\mathrm{BN}$ nano-crystals and may attract further attention of the experimental scientists in future.

\section{References}

1. P. B. Mirkarimi, K. F. McCarty, D. L. Medlin, Materials Science and Engineering, R21, 47 (1997).

2. M. P. Surh, S. G. Louie, M. L. Cohen, Phys. Rev. B 43, 9126 (1991).

3. S. C. Vanithakumari, and K. K. Nanda, J. Phys. Chem. B 110, 1033 (2006).

4. W. H. Qi, M. P. Wang, M. Zhou, and W. Y. Hu, J. Phys. D: Appl. Phys. 38, 1429 (2005).

5. Chang Q. Sun, H. L. Bai, S. Li, B. K. Tay, C. Li, T. P. Chen, and E. Y. Jiang, J. Phys. Chem. B 108, 2162 (2004).

6. M. Wautelet, J. P. Dauchot, and M. Hecq, J. Phys.: Condens. Matter 15, 3651 (2003).

7. Xiaotian Li, Changlu Shao, Shilun Qiua, Feng-Shou Xiao, Weitao Zheng, Pinliang Ying, Osamu Terasaki, Microporous and Mesoporous Materials 40, 263-269 (2000).

8. Takeo Oku, Masaki Kuno, Synthesis, Diamond and Related Materials 12, 840-845 (2003).

9. L. Liu, Y. P. Feng, and Z. X. Shen, Phys. Rev. B 68, 104102 (2003).

10. Yongqing Cai, Litong Zhang, Qingfeng Zeng, Laifei Cheng, Yongdong Xu, Solid State Communications 141, 262-266 (2007).

11. Elise Knittle, Renata M. Wentzcovitch, Reymond Jeanloz and L. Cohen, Nature 337, 26 (1989).

12. A. N. Goldstein, C. M. Esher, and A. P. Alivisatos, Science 256, 1425 (1992).

13. H. H. Farrell, C. D. Van Siclen, J. Vac. Sci. Technol. B 25, 1441 (2007). 